Wien klin Mag 2020 - 23:288-296 https://doi.org/10.1007/s00740-020-00371-z Online publiziert: 25 . November 2020 (c) Der/die Autor(en) 2020

\section{Susanne M. Lang · Tobias Rachow}

Klinik für Innere Medizin II, Sektion Pneumologische Onkologie, Universitätsklinikum Jena, Jena, Deutschland

\title{
Molekulare therapeutische Stratifizierung des nichtkleinzelligen Lungenkarzinoms
}

Die aktuellen Fortschritte bei der Behandlung des nichtkleinzelligen Lungenkarzinoms (NSCLC) sind beeindruckend und im Wesentlichen durch die Einführung der Immuntherapie geprägt. Zielgerichtete Therapien haben ihre Bedeutung für Subgruppen von Patienten mit Treibermutationen jedoch nicht verloren. Neue Treibermutationen mit neuen medikamentösen Optionen stellen den behandelnden Arzt und Pathologen vor die Herausforderung, molekulare Targets kurzfristig zu analysieren, um die bestmögliche Erstlinien- und Folgetherapie festzulegen. Im Folgenden werden die molekular stratifizierten therapeutischen Optionen beim NSCLC dargestellt.

\section{Zielgerichtete Therapien bei Treibermutationen}

Mit der Einführung zielgerichteter Therapien für Patienten mit Epidermal-Growth-Factor-Receptor(EGFR)oder B-Rapidly-Accelerated-Fibrosarcoma(BRAF)-p.V600E-Mutationen, Anaplastic-Lymphoma-Kinase( $A L K)$ - oder ROS-Proto-Oncogene-1(ROS1)-Translokationen oder Mesenchymal-Epithelial-Transition(MET)-Amplifikationen konnte im Mittel eine Verlängerung des Gesamtüberlebens (OS) von etwa 1,5 Jahren erreicht werden gegenüber

Dieser Beitrag wurde in der Zeitschrift Der Onkologe 8 (2020) 26:727-735. https://doi.org/ 10.1007/s00761-020-00787-6 erstpubliziert. Zweitpublikation mit freundlicher Genehmigung der Autoren.
Patienten, die nicht mit zielgerichteten Therapien behandelt wurden [1]

Seither ist es Standard, eine $\mathrm{Mu}$ tationsanalyse durchzuführen, um die Option einer individuellen zielgerichteten Therapie durch Tyrosinkinaseinhibitoren (TKI) zu prüfen. Dies gilt auch im Zeitalter der Immuntherapie, da treibermutierte nichtkleinzellige Lungenkarzinome (NSCLC) schlechter auf Immuntherapien ansprechen oder es sogar zu einer Hyperprogression kommen kann. Aus den Analysen des Cancer Genome Atlas geht hervor, dass eine inverse Korrelation zwischen EGFR-Mutation und PD-L1-Expression besteht. Neben der PD-L1-Expression gelten als weitere prognostische Faktoren für ein Ansprechen der Immuntherapie der Raucherstatus und die Tumormutationslast (TMB, „tumor mutational burden“). Patienten mit EGFR-Mutationen sind jedoch häufig Nie- oder Leichtraucher, und die Tumoren haben nur eine geringe TMB [9]. In der Metaanalyse von Lee et al. wurden 5 randomisierte Studien mit verschiedenen Checkpointinhibitoren in der zweiten Therapielinie im Vergleich zur Standardtherapie mit Docetaxel analysiert. In der Subgruppe von Patienten mit EGFR-Mutationen fand sich ein schlechteres Outcome vs. Docetaxel (Hazard Ratio, HR, für OS: 1,11; 95\%Konfidenzintervall, 95\%-KI: 0,80-1,53) [21].

Die aktuelle deutsche S3-Leitlinie empfiehlt, vor Einleitung der Erstlinientherapie als Mindestanforderung EGFRMutationen in den Exonen 18-21, ALK-
Fusionen und ROS1-Fusionen, BRAFV600-Mutationen, innerhalb von $10 \mathrm{Ar}-$ beitstagen bei nichtplattenepithelialen NSCLC zu bestimmen. Dies gilt ebenfalls für Plattenepithelkarzinome von Nierauchern oder Leichtrauchern [20].

\section{Treibermutationen mit den meisten Behandlungsoptionen}

Aktivierende EGFR-Mutationen und $A L K$-Translokationen sind aktuell die beiden molekularen Treibermutationen mit den meisten Behandlungsoptionen.

\section{Aktivierende EGFR-Mutationen}

Der EGF-Rezeptor ist ein Transmembranmolekül aus der Human-EpidermalGrowth-Factor-Receptor(HER)-Wachstumsfaktor-Genfamilie. Das EGFR-Molekül besteht aus einer extrazellulären Ligandenbindungs- und einer intrazellulären Tyrosinkinasedomäne. Nach Bindung an einen passenden Liganden formieren sich Homo- oder Heterodimere, die zur Autophosphorylierung des Moleküls führen und den Signalweg aktivieren. Mutationen in der Tyrosinkinasedomäne des EGF-Rezeptors führen zu einer ligandenunabhängigen Aktivierung der EGF-Rezeptor-Signalkaskade und verschaffen der Tumorzelle einen Überlebensvorteil (• Abb. 1).

In Europa weisen etwa $12-20 \%$ der pulmonalen Adenokarzinome aktivierende EGFR-Mutationen auf. Im asiatischen Raum können bis zu $48 \%$ der Adenokarzinome Mutationen aufwei- 




Abb. $1 \Delta$ a Schematische Darstellung der Aktivierung des Epidermal-Growth-Factor-Rezeptors (EGFR) durch Ligandenbindung oder aktivierende Mutation mit nachfolgenden Signalwegen; $\mathbf{b}$ Inhibition der Bindung von Adenosintriphosphat (ATP) durch Tyrosinkinaseinhibitoren (TKI)

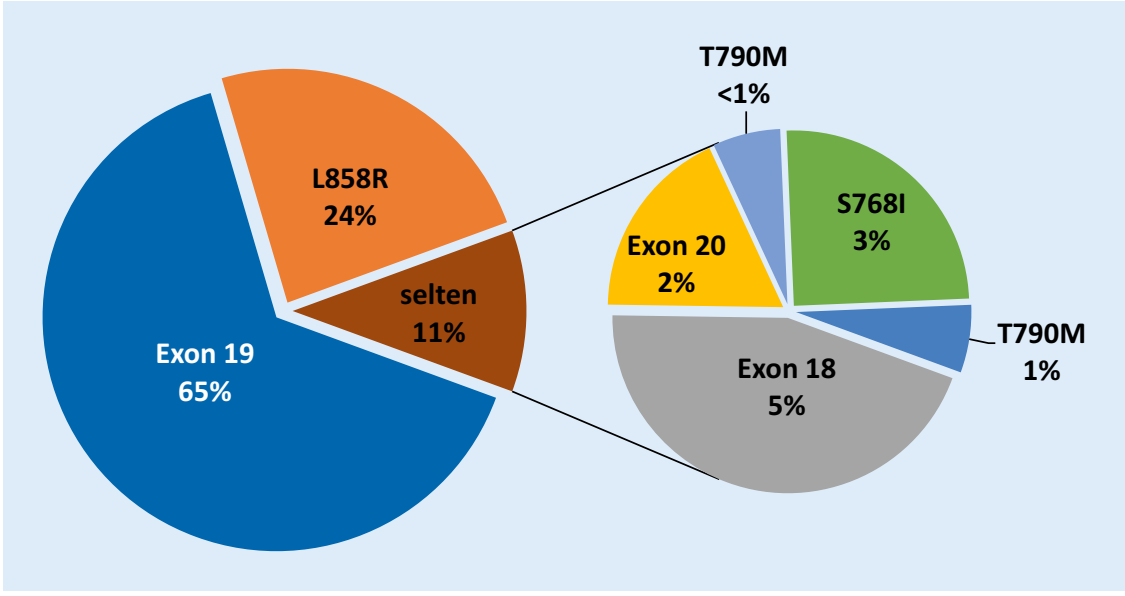

Abb. $2 \Delta$ Häufigkeiten ausgewählter aktivierender EGFR-Mutationen (Epidermal-Growth-Factor-Rezeptor) in Europa. (Mod. nach Graham et al., 2018 [13])

sen. Die Mutationen in Exon 19 sowie die L858R-Mutation werden als häufige Mutationen, d.h. „common mutations“, die seltener auftretenden Mutationen als „uncommon mutations“ bezeichnet (• Abb. 2; [13]).

Die nachgewiesenen Mutationen des EGFR-Gens sprechen z. T. unterschiedlich auf die Blockade durch TKI an. We- sentliche, klinisch relevante Mutationen beim NSCLC sind in $\bullet$ Tab. 1 dargestellt.

\section{Klinische Besonderheiten}

EGFR-Mutationen treten häufiger bei Nicht- oder Leichtrauchern, Frauen oder Patienten mit asiatischer Herkunft auf [13].

\section{Molekulare Diagnostik}

Mutationen im EGFR-Gen werden durch Sequenzierungstechniken der Exons 18-20 des EGFR-Gens detektiert. Neben der Diagnostik im Tumormaterial ist auch eine Mutationsanalyse aus der zirkulierenden Tumor-DNA in einer „liquid biopsy“ möglich. Diese Methode erfordert lediglich eine Blutentnahme und ermöglicht den Nachweis von Mutationen, Deletionen und Amplifikationen aus zirkulierenden Tumorzellen („,circulating tumor cells", CTC) und aus zellfreier DNA („cell-free DNA“, cfDNA), die aus Tumorzellen in den Blutstrom abgegeben wird.

》) Die Trefferquote der „liquid biopsy" ist niedriger als bei Biopsien, doch die Spezifität ist hoch

Im Gegensatz zu Biopsiematerial ist die Trefferquote der „liquid biopsy“ zwar niedriger, die Spezifität ist jedoch hoch. Im ambulanten Setting ist die Testung 


\section{S. Lang $\cdot$ T. Rachow}

\section{Molekulare therapeutische Stratifizierung des nichtkleinzelligen Lungenkarzinoms}

\section{Zusammenfassung}

Hintergrund. Die Therapiemöglichkeiten des nichtkleinzelligen Lungenkarzinoms (NSCLC) im Stadium IV haben sich in den letzten Jahren rasant entwickelt. Das Überleben der Patienten wurde durch die zielgerichtete Behandlung von Tumoren mit EGFR-, BRAF-Mutationen, EML4/ALK- und ROS1-Translokationen verbessert. Durch die Entwicklung neuer Therapieoptionen wird die molekulare Stratifizierung der Therapie für den behandelnden Arzt immer schwieriger. Ziel der Arbeit. Die Übersicht gibt einen aktuellen Überblick über die molekular stratifizierten therapeutischen Optionen beim NSCLC.

Ergebnisse. Durch reversible EGFR-Tyrosinkinaseinhibitoren (TKI) ließ sich das Überleben von NSCLC-Patienten mit EGFR-Mutation verlängern. Im Verlauf kommt es jedoch unweigerlich zur Resistenzentwicklung, die durch irreversible EGFR-TKI z. T. überwunden werden konnte. Aktuelle Studien zeigen einen möglichen Vorteil der Kombination von TKI mit Angiogeneseinhibitoren oder Chemotherapie. Bei EML4-/ALK- (und ROS1-)positiven NSCLC war der Multityrosinkinaseinhibitor Crizotinib als Erstes verfügbar. Potentere Zweitgenerations-ALK-TKI vermochten die Crizotinibresistenz zu durchbrechen. Mit der Einführung eines hochpotenten Drittgenerations-TKI konnten weitere ALKResistenzen überwunden werden. Für BRAFHER2-, MET-Exon-14-Skipping und RETMutationen stehen zielgerichtete Substanzen zur Verfügung, die z. T. bereits zugelassen sind. Larotrectinib erhielt als erste Substanz in der EU eine tumorunabhängige Zulassung für NTRK-positive Tumoren.

Schlussfolgerung. Die Weiterentwicklung zielgerichteter Therapien erfordern die molekulare Analyse des Tumorgewebes und molekulare Stratifizierung der Therapie. Unklar bleiben die optimale Therapiesequenz und mögliche Verbesserungen durch Kombinationstherapien.

\section{Schlüsselwörter}

Zelloberflächenrezeptoren · Anaplastische Lymphomkinase - Protein-Tyrosinkinase . EGFR-Protein, humanes · Protoonkogenproteine

\section{Molecular treatment stratification of non-small cell lung cancer}

\section{Abstract}

Background. Treatment modalities for stage IV non-small cell lung cancer (NSCLC) have vastly improved over the last few years. Targeted therapies of tumours with EGFR and BRAF mutations, EMLA/ALK and ROS1 rearrangements offer improved survival. New targets are being studied and it is increasingly demanding for the treating physician to decide which therapy best fits the individual molecular profile of the tumour.

Objectives. The review offers an up-to-date survey of current molecular targets and treatment options.

Results. First-generation reversible EGFR tyrosine kinase inhibitors (TKI) have led to improved survival in patients with EGFRmutated tumours, but resistance invariably occurs with continuing treatment. Irreversible EGFR TKIs have been developed to counter such resistance. Recent studies indicate further improvements by combining TKIs with angiogenesis inhibitors or chemotherapy. In the case of EML4/ALK- (and ROS1)-positive NSCLC, crizotinib, a multitarget TKI was the first available treatment choice; meanwhile some more potent second-generation ALK TKIs have been developed to overcome crizotinib resistance and recently a potent and highly selective third-generation inhibitor was introduced to overcome known $A L K$ resistance mutations. BRAF, MET exon 14 skipping, HER2 and RET mutations also have viable treatment options and larotrectinib received a tissue- agnostic EU approval for tumours bearing NTRK fusions.

Conclusion. The wealth of therapeutic options for the treatment of druggable molecular targets makes a molecular workup of the tumour and treatment guided by molecular targets mandatory. The optimal sequence of therapy and possible improvement by combinations with chemotherapy or angiogenesis inhibitors remain a matter of debate.

\section{Keywords}

Cell surface receptors - Anaplastic lymphoma kinase $\cdot$ Protein-tyrosine kinases · EGFR protein, human . Proto-oncogene proteins auf EGFR-Mutationen seit 2018 erstattungsfähig. Im klinischen Alltag hat die "liquid biopsy“ einen besonderen Stellenwert (a) bei Patienten, denen eine invasive Prozedur nicht zugemutet werden kann, oder (b) bei ungünstiger Lage des Tumors und (c) im Verlauf bei wiederholten Untersuchungen auf Resistenzmutationen.

\section{Therapieoptionen}

Die Erstgenerationspräparate Erlotinib und Gefitinib binden reversibel an die
Tyrosinkinasedomäne des EGFR-Moleküls. Zweitgenerations-TKI, wie Afatinib oder Dacomitinib, hingegen entfalten ihre Wirkung durch eine irreversible Bindung (-Tab. 2). Letztlich resultiert die Blockade einer Bindungsstelle für Adenosintriphosphat (ATP), wodurch die Aktivierung der nachfolgenden Signalkaskade verhindert wird. Afatinib hat zudem eine deutlich höhere Affinität zur Kinasedomäne als die Substanzen der ersten Generation. Klinisch übersetzte sich dies in der LUX-Lung-7-Studie in ein deutlich längeres progressionsfreies Überleben (PFS) bei der Behandlung mit Afatinib im Vergleich zu einer Therapie mit Gefitinib [32]. Dacomitinib bindet auch an weitere HER-Moleküle wie „erythroblastic oncogene B 2“ (ERBB2) und ERBB4. In der ARCHER-1050Studie wurde die klinische Effektivität von Dacomitinib im Vergleich zu Gefitinib bei Patienten ohne Hirnmetastasen untersucht. Es zeigten sich auch hier signifikante Vorteile, sowohl für das PFS als auch für das OS [25]. 


\begin{tabular}{|c|c|c|}
\hline Exon & Art der Mutation & $\begin{array}{l}\text { Ansprechen } \\
\text { auf TKI }\end{array}$ \\
\hline \multicolumn{3}{|c|}{$\begin{array}{l}\text { Typische Mutationen („,common mutati- } \\
\text { ons") }\end{array}$} \\
\hline 19 & Deletion & $\begin{array}{l}\text { Alle zugelas- } \\
\text { senen TKI }\end{array}$ \\
\hline 21 & L858R-Mutation & $\begin{array}{l}\text { Alle zugelas- } \\
\text { senen TKI }\end{array}$ \\
\hline \multicolumn{3}{|c|}{$\begin{array}{l}\text { Seltene Mutationen („uncommon mutati- } \\
\text { ons") }\end{array}$} \\
\hline 18 & G719X & $\begin{array}{l}\text { Alle zugelas- } \\
\text { senen TKI }\end{array}$ \\
\hline 19 & Insertion & $\begin{array}{l}\text { Alle zugelas- } \\
\text { senen TKI }\end{array}$ \\
\hline \multirow[t]{4}{*}{20} & $\begin{array}{l}\text { S768I } \\
\text { C797S }\end{array}$ & $\begin{array}{l}\text { Alle zugelas- } \\
\text { senen TKI }\end{array}$ \\
\hline & T790M & Osimertinib \\
\hline & A763_Y764insFQEA & $\begin{array}{l}\text { Alle zugelas- } \\
\text { senen TKI }\end{array}$ \\
\hline & $\begin{array}{l}\text { Andere } \\
\text { Exon-20-Insertionen }\end{array}$ & $\begin{array}{l}\text { Schlechtes } \\
\text { Ansprechen }\end{array}$ \\
\hline 21 & L861Q & $\begin{array}{l}\text { Alle zugelas- } \\
\text { senen TKI }\end{array}$ \\
\hline
\end{tabular}

Der Drittgenerations-EGFR-TKI Osimertinib bindet ebenfalls kovalent und irreversibel an seine Zielstruktur, unterscheidet sich jedoch von den anderen Substanzen v. a. durch eine höhere Affinität zu mutierten EGFR-Molekülen. Nachdem die Substanz zunächst in der Zweitlinie bei Nachweis der T790M-Mutation zugelassen wurde, konnte seine Überlegenheit auch in der Erstlinie gezeigt werden. Im Vergleich zu Gefitinib oder Erlotinib ergaben sich unter Osimertinib signifikante Vorteile sowohl für das PFS als auch für das OS [37]. Aufgrund dieser Daten wird der Einsatz von Osimertinib in der Erstlinientherapie empfohlen. In der aktuellen Onkopedia-Leitlinie werden Osimertinib oder Dacomitinib oder Afatinib empfohlen. Vergleichende Daten von Dacomitinib gegenüber Osimertinib oder Afatinib liegen nicht vor [2].

Mit Ausnahme der Exon-20-Insertionen sprechen auch die selteneren Mutationen (•Tab. 1) in der Regel gut auf EGFR-TKI an, wobei eine Behandlung mit Zweit- oder DrittgenerationsTKI einen besseren Therapieerfolg ver-

\begin{tabular}{ll|l|l|}
\hline Tab. 2 & Charakteristika aktuell verfügbarer Tyrosinkinaseinhibitoren (TKI) \\
& Erstgenerations-TKI & $\begin{array}{l}\text { Zweitgene- } \\
\text { rations-TKI }\end{array}$ & Drittgenerations-TKI \\
\hline Charakteristika & $\begin{array}{l}\text { Reversible Bindung im Bereich } \\
\text { der Tyrosinkinasedomäne }\end{array}$ & $\begin{array}{l}\text { Irreversible } \\
\text { Bindung }\end{array}$ & $\begin{array}{l}\text { Überwindung der } \\
\text { T790M-Mutation mög- } \\
\text { lich }\end{array}$ \\
\hline $\begin{array}{l}\text { Zugelassene } \\
\text { Substanzen }\end{array}$ & $\begin{array}{l}\text { Erlotinib (+Bevacizumab) } \\
\text { Gefitinib }\end{array}$ & $\begin{array}{l}\text { Afatinib } \\
\text { Dacomitinib }\end{array}$ & Osimertinib \\
\hline
\end{tabular}

spricht als eine Behandlung mit Erlotinib oder Gefitinib [45].

Nahezu alle Insertionen im Exon 20 (- Tab. 1) sollten, aufgrund der schlechten Wirksamkeit der bisher einsetzbaren TKI, analog zu Tumoren ohne nachgewiesene Treibermutation behandelt werden.

Monoklonale EGFR-Antikörper wie beispielsweise Cetuximab sind gegen die extrazellulären Anteile des EGF-Rezeptors gerichtet und verhindern durch ihre Bindung die ligandenabhängige Rezeptoraktivierung. Sie spielen bei der Behandlung von Lungenkarzinomen keine Rolle.

\section{Kombinationstherapien}

Die Kombination von EGFR-TKI mit anderen Formen der Systemtherapie wurde in aktuellen Studien untersucht. Kürzlich wurde in einer japanischen Arbeit gezeigt, dass eine Behandlung mit Gefitinib und Carboplatin/Pemetrexed im Vergleich zur Chemotherapie zu verbesserten Ansprechraten führt [16]. Dies galt auch für eine indische Studie mit 350 Patienten, in der unter der Kombination von Gefitinib mit Carboplatin/ Pemetrexed gegenüber der alleinigen Chemotherapie ein verlängertes Überleben erreicht wurde [27].

Auch die duale Blockade des EGFRund Vascular-Endothelial-Growth-Factor(VEGF)-Signalwegs durch Erlotinib plus Ramucirumab hat vielversprechende Ergebnisse bzgl. des PFS gezeigt [26].

\section{Resistenzentwicklung}

Eine Behandlung mit EGFR-TKI führt, auch bei zunächst gutem Ansprechen, in der Regel im weiteren Verlauf zur Krankheitsprogression. Eine wesentliche Ursache hierfür sind resistenzvermittelnde Mutationen, die im Median nach etwa 12 Monaten auftreten. Bei einem Rezidiv bzw. Progress der Erkrankung sollte eine Rebiopsie angestrebt werden, um auf entsprechende Mutationen zu testen. Alternativ kann die EGFR-T790M-Mutationsanalyse über die "liquid biopsy“ erfolgen [29].

T790M-Mutation. Die häufigste resistenzvermittelnde Mutation, die in etwa $50 \%$ der sekundären EGFR-TKI-Resistenzen nachgewiesen werden konnte, ist die T790M-Punktmutation, welche über den Austausch von Methionin gegen Threonin an der Position $790 \mathrm{zu}$ einer verstärkten ATP-Bindungsaffinität und damit zu einem Wirkverlust der Erstund Zweitgenerations-EGFR-TKI führt [31]. In der AURA-Studie wurde gezeigt, dass der Drittgenerations-EGFRTKI Osimertinib diesen Resistenzmechanismus erfolgreich überwinden kann [12].

C797S-Mutation. Die resistenzvermittelnde C797S-Mutation führt $\mathrm{zu}$ einem Aminosäureaustauch von Cystein zu Serin in der Position 797 auf dem Exon 20. Die damit verbundene Strukturänderung des EGF-Rezeptors verhindert eine Bindung von Erst-, Zweit- und auch Drittgenerations-EGFR-TKI. Diese Mutation konnte in TKI-naiven Tumoren nicht nachgewiesen werden und wird erst im Laufe der Behandlung mit einem TKI erworben [28]. Aktuell befinden sich allosterische EGFR-Inhibitoren zur Überwindung der C797S-Mutation in der Entwicklung [17].

Bei Versagen der zielgerichteten Therapie mit EGFR-TKI kommt eine Immunchemotherapie infrage

Weitere EGFR-unabhängige Resistenzmechanismen, die zu einem Versagen der 


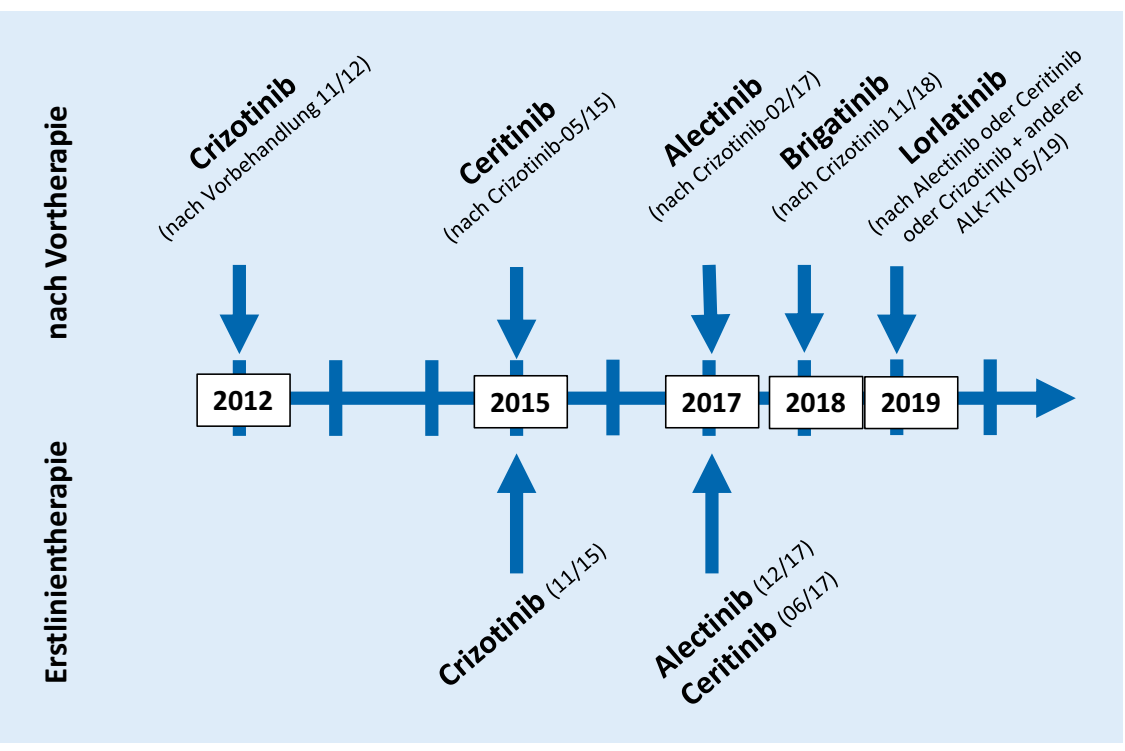

Abb. $3 \Delta$ Zeitlicher Verlauf von Neuzulassungen von ALK-Tyrosinkinaseinhibitoren (Anaplastic-Lymphoma-Kinase-TKI) in der Erstlinientherapie oder nach erfolgter Vorbehandlung durch die European Medicines Agency (EMA)

EGFR-gerichteten Therapie führen, sind MET- und HER2/neu-Amplifikationen, PI3-Kinase-Mutationen oder eine Transformation in ein kleinzelliges Karzinom.

Bei Versagen einer zielgerichteten Therapie mit EGFR-TKI kann eine Immunchemotherapie erfolgreich eingesetzt werden. In der IMpower-150Studie wurden Patienten mit EGFRMutation oder $A L K$-Translokation nach ausgeschöpfter TKI-Therapie mit einer Kombination von Atezolizumab, Carboplatin, Paclitaxel und Bevacizumab behandelt. Es zeigte sich ein verbessertes Gesamtüberleben mit einer HR von 0,61 (95\%-KI: 0,29-1,28) [39]. Diese Therapie kann nach TKI-Versagen eingesetzt werden.

\section{EML4/ALK-Translokationen}

Onkogene Fusionsmoleküle der anaplastischen Lymphomkinase $(A L K)$ wurden 2007 erstmals bei Lungenkarzinomen nachgewiesen [42]. Der häufigste Fusionspartner ist EML4 („echinoderm microtubule-associated protein-like $4^{\prime \prime}$ ), und die meisten Fusionen resultieren aus einer Inversion auf dem kurzen Arm des Chromosoms 2. Bei etwa 5\% der NSCLC (überwiegend Adenokarzinome) können $A L K$-Translokationen nachgewiesen werden.

\section{Klinische Besonderheiten}

Patienten mit einer ALK-Translokation sind oftmals jünger, häufiger Nicht- oder Leichtraucher und befinden sich zum Zeitpunkt der Diagnosestellung bereits in einem fortgeschrittenen Krankheitsstadium (besonders Leber-, Hirnmetastasen, Pleura- oder Perikardergüsse) [5]. Die Gruppe um Rangachari et al. verfolgte eine Kohorte von 21 Patienten mit $A L K$-Translokation über 4 Jahre und konnte eine kontinuierliche $\mathrm{Zu}$ nahme der Hirnmetastasen von 23,8\% im 1. Jahr auf $45,5 \%$ im 2. Jahr und $58,4 \%$ im 3. Jahr nachweisen [38]. Eine weitere Besonderheit ist das erhöhte Risiko thrombembolischer Ereignisse, mit einer 3- bis 5-fach erhöhten Inzidenz und schwerem Verlauf [44].

\section{Molekulare Diagnostik}

Die FISH-Analyse gilt als Goldstandard in der Diagnostik von ALK-Translokationen bei Patienten mit pulmonalem Adenokarzinom, da $A L K$-Fusionen jedoch auch zu einer verstärkten Proteinexpression an der Zelloberfläche führen, ist eine Analyse mittels Immunhistochemie (IHC) ebenfalls möglich.

\section{Therapieoptionen}

Der Multityrosinkinaseinhibitor Crizotinib, der ursprünglich als MET-Inhibitor entwickelt wurde, führte $\mathrm{zu}$ einem gu- ten Therapieansprechen bei EML4/ALKtranslozierten Lungenkarzinomen. In klinischen Studien wurde gezeigt, dass eine zielgerichtete Therapie mit Crizotinib bei Vorhandensein einer ALKTranslokation der klassischen Chemotherapie sowohl in der Erst-, aber auch in der Zweitlinientherapie deutlich überlegen ist $[41,43]$. Dies galt jedoch nicht für Patienten mit Metastasen im zentralen Nervensystem (ZNS).

Der Zweitgenerations ALK-TKI Alectinib weist eine bessere Wirksamkeit im ZNS auf und ist Crizotinib im Hinblick auf das PFS signifikant überlegen [33]. Alectinib ist derzeit die Standard-Erstlinientherapie bei metastasiertem NSCLC mit einer EML4/ALK-Translokation [36]. Auch für weitere Zweitgenerations-ALKInhibitoren wie Ceritinib oder Brigatinib konnte eine gute Wirksamkeit im ZNS gezeigt werden.

Der Next-Generation-TKI Lorlatinib ist ein reversibler ATP-kompetitiver makrozyklischer TKI mit dem breitesten Wirkspektrum der aktuell zugelassenen ALK-TKI. Bei unvorbehandelten Patienten betrug die Ansprechrate $90 \%$. Lorlatinib ist auch nach Vorbehandlung mit Crizotinib, Alectinib, Brigatinib oder Ceritinib wirksam [40].

Unterdessen sind für diese seltene Subgruppe von Patienten mit $A L K$ Translokation eine Reihe von TKI zugelassen (• Abb. 3).

\section{Resistenzentwicklung}

Nach etwa 1-2 Jahren tritt üblicherweise eine Resistenz gegenüber dem ALK-TKI Crizotinib auf. Resistenzvermittelnd sind sog. On-Target-Mechanismen (sekundäre Mutationen in der ALK-Tyrosinkinasedomäne, Fusionsgenamplifikationen) oder Off-Target-Mechanismen (Hochregulation alternativer onkogener Signalwege, z. B. EGFR-, IGF-, RAS/SRC-, AKT/mTOR-Signalwege). Anhand des resistenzvermittelnden Mechanismus kann die weitere Therapie geplant werden. Beispielsweise können eine duale ALK- und EGFR-Inhibition oder eine duale ALK- und KIT-Inhibition bei bestehender EGFR-Aktivierung oder KITAmplifikation erfolgversprechend sein, sind aber noch nicht zugelassen [19]. 
Die Art der resistenzvermittelnden Mutation hat eine entscheidende Bedeutung. Codon-1196-Mutationen vermindern die Kinase-ATP-Interaktion von Crizotinib. Dieser Resistenzmechanismus kann jedoch durch potentere ALKInhibitoren überwunden werden.

Im Gegensatz dazu führen im Verlauf auftretende Mutationen im Codon 1202 zu einer Resistenz gegenüber den meisten verfügbaren ALK-Inhibitoren mit Ausnahme von Lorlatinib. Die G1202R-Mutation macht nach Versagen einer Therapie mit Zweitgenerations-TKI (Standardtherapie mit Alectinib) den größten Anteil resistenzvermittelnder Mutationen aus [11].

\section{》) Die Art der resistenzver- mittelnden Mutation hat eine entscheidende Bedeutung}

Angesichts der verschiedenen bekannten Resistenzmechanismen und der damit verbundenen unterschiedlichen Sensitivität gegenüber den aktuell zugelassenen Inhibitoren sollte vor einer Therapieumstellung eine Rebiopsie zur erneuten molekularen Testung des Tumormaterials angestrebt werden.

\section{Weitere molekulare Treiber mit zugelassenen TKI}

Aktivierende molekulare Treibermutationen/Genfusionen mit der Option einer zielgerichteten Therapie konnten in den Genen BRAF, ROS1 und NTRK nachgewiesen werden.

\section{Translokationen von ROS1}

Bei etwa 1-2\% der Lungenkarzinome (Adenokarzinome) können ROS1-Genfusionen nachgewiesen werden.

ROS1 ist eine Rezeptortyrosinkinase, die Strukturähnlichkeiten mit ALK aufweist. Es sind viele mögliche Fusionspartner (z.B. CD74, SLC42A2, EZR oder FIG1) bekannt. Vermutlich beruht die onkogene Wirkung auf der Aktivierung von PI3-Kinase-, STAT3- und RAS/ MAPK-Signalwegen.

\section{Klinische Besonderheiten}

Der klinische Phänotyp ähnelt dem von Patienten mit ALK-Translokationen. Patienten, bei denen eine ROS1-Translokation nachgewiesen werden konnte, sind meist jünger und haben nie oder kaum geraucht. In einer großen Fallsammlung von 257 Patienten mit Treibermutationen (ROS1, $n=53 ; A L K, n=87$; EGFR, $n=117)$ wurde gezeigt, dass bei ROS1positiven Patienten weniger häufig Hirnmetastasen (ROS1 9\%; ALK 25\%; EGFR $40 \%$; $p<0,04)$ und extrathorakale Metastasen (ROS1 49\%; ALK $75 \%$; EGFR $72 \% ; p<0,01)$, jedoch häufiger Lymphknotenmetastasen (ROS1, 15\%; EGFR, $2 \% ; p<0,01)$ und sklerosierende Knochenmetastasen (ROS1, 17\%; EGFR, $6 \% ; p<0,01)$ auftraten [8]. Die Gruppe um Chiari konnte in einer prospektiven Phase-II-Studie zur Wirksamkeit von Crizotinib zeigen, dass das Risiko thromboembolischer Ereignisse bei 48 Patienten mit ROS1-Translokation um $14 \%$ höher war als bei den anderen Patienten mit Treibermutationen [6].

\section{Molekulare Diagnostik}

ROS1-Translokationen können mittels Fluoreszenz-in-situ-Hybridisierung (FISH) in der Routine nachgewiesen werden (Goldstandard). Angesichts der niedrigen Frequenz des Auftretens der Veränderung hat es sich in der Praxis jedoch bewährt, zunächst eine immunhistochemische Testung und nachfolgend eine FISH-Analyse oder andere molekulare Methoden als Bestätigungstest durchzuführen. Die Leitlinien empfehlen die ROS1-Testung bei allen Patienten mit fortgeschrittenem Adenokarzinom der Lunge, unabhängig von klinischen Charakteristika [18].

\section{Therapie}

In klinischen Studien wurde gezeigt, dass Crizotinib - ein MultikinaseMET/ALK/ROS1-TKI - auch Wirksamkeit bei ROS1-translozierten Lungenkarzinomen hat [23]. In einer PhaseI-Studie fand sich ein objektives Ansprechen von $72 \%$ und ein medianes PFS von 19,2 Monaten. Momentan ist Crizotinib der Standard in der Erstlinientherapie.

Unter der Behandlung mit Lorlatinib wurde bei 6 von 12 Patienten ein partiel- le Remission und bei 2 weiteren Krankheitskontrolle erreicht - mit einer mittleren Dauer des Ansprechens von $12 \mathrm{Mo-}$ naten (95\%-KI: 5,7-NR, nicht erreicht) [33].

Entrectinib ist ein Multikinase-TRKA-, TRKB-, TRKC-, ROS1- und ALK-TKI. Bei 51 Erwachsenen mit NSCLC und ROS1-Translokation, die mit Entrectinib behandelt wurden, zeigte sich eine objektive Ansprechrate (ORR) von 78\%. Die Dauer des Ansprechens lag bei $55 \%$ der Patienten bei 12 Monaten oder mehr. Aufgrund dieser Ergebnisse hat die US-amerikanische Food and Drug Administration (FDA) in Bezug auf Entrectinib grünes Licht für die Therapie des metastasierten ROS1-positiven NSCLC gegeben [14].

In Deutschland ist aktuell nur Crizotinib für die Behandlung des fortgeschrittenen ROS1-positiven NSCLC zugelassen.

\section{BRAF-Onkogen}

In der Literatur werden Mutationen im $B R A F-G e n$ mit einer Häufigkeit von 1-4\% der Lungenkarzinome vom Adenokarzinomtyp angegeben.

\section{》) Sowohl aktivierende als auch inaktivierende Mutationen tragen paradoxerweise zum Tumorwachstum bei}

BRAF ist eine Serin-/Threoninkinase im RAS-RAF-MEK-ERK-Signalweg. Am häufigsten findet sich der Austausch einer Glutaminsäure durch Valin an der Position 600 (BRAF V600E) in der Proteinkinasedomäne. Aktivierende Mutationen führen über eine Phosphorylierung von $M E K$ durch $B R A F$ letztlich zum Zellwachstum, zur Proliferation und zum Überleben von Tumorzellen. Seltener kommen inaktivierende Mutationen (z.B. BRAF G466V oder BRAF Y472C) im BRAF-Gen vor. Sowohl aktivierende als auch inaktivierende Mutationen tragen paradoxerweise zum Tumorwachstum bei. Im Gegensatz zum malignen Melanom findet man beim NSCLC in weniger als $50 \%$ der Fälle die V600E-Mutation. Die zweithäufigste 


\begin{tabular}{|c|c|c|c|}
\hline Gen & Mutation & Therapieoptionen & Literatur \\
\hline \multirow[t]{2}{*}{ NTRK } & \multirow{2}{*}{$\begin{array}{l}\text { Fusion der } \\
\text { Tyrosinkinasedomäne mit } \\
\text { einem von mehr als } \\
50 \text { bekannten } \\
\text { Fusionspartnern }\end{array}$} & Larotrectinib $^{a}$ & \multirow[t]{2}{*}{ Guo [14] } \\
\hline & & Entrectinib & \\
\hline \multirow[t]{4}{*}{ MET } & \multirow{4}{*}{$\begin{array}{l}\text { Exon-14-Skipping } \\
\text { Amplifikation }\end{array}$} & Crizotinib & \multirow{4}{*}{$\begin{array}{l}\text { Drilon } \\
{[10]}\end{array}$} \\
\hline & & Capmatinib & \\
\hline & & Tepotinib & \\
\hline & & Savolitinib & \\
\hline \multirow[t]{2}{*}{ KRAS } & \multirow[t]{2}{*}{$\mathrm{G} 12 \mathrm{C}$} & MRTX849 & \multirow{2}{*}{$\begin{array}{l}\text { Dafna } \\
\text { Bar-Sagi } \\
{[4]}\end{array}$} \\
\hline & & AMG 510 & \\
\hline \multirow[t]{3}{*}{ ERBB2/HER2 } & \multirow{3}{*}{$\begin{array}{l}\text { HER2/ERBB2-Amplifikationen } \\
\text { und HER2/ERBB2-Mutationen }\end{array}$} & Afatinib & \multirow[t]{3}{*}{ Pillaj [34] } \\
\hline & & Neratinib & \\
\hline & & Lapatinib & \\
\hline $\begin{array}{l}\text { MAP2K1 } \\
\text { (MEK1) }\end{array}$ & $15 q 22.31$ & $\begin{array}{l}\text { ERK1/2-Inhibitor LY3214996 } \\
\text { Vistusertib } \\
\text { Abemaciclib }\end{array}$ & Arcila [3] \\
\hline FGFR1 & $\begin{array}{l}\text { Amplifikation } \\
\text { Mutation }\end{array}$ & $\begin{array}{l}\left.\text { Nintedanib plus Docetaxel ( }{ }^{\mathrm{a}} 2 \mathrm{~L}\right) \\
\text { Dovitinib }\end{array}$ & Guo [14] \\
\hline $\begin{array}{l}\text { RET-Re- } \\
\text { arrangement }\end{array}$ & Chromosom 10q11.2 & $\begin{array}{l}\text { Cabozantinib, Vandetanib } \\
\text { Selpercatinib }\end{array}$ & Guo [14] \\
\hline \multicolumn{3}{|c|}{ a Zugelassen, $1 \mathrm{~L}$ (Erstlinientherapie) } & \\
\hline
\end{tabular}

Mutation beim Lungenkarzinom ist die G469A-Mutation (39\%). Weitere NonV600-Mutationen finden sich in einigen Hotspots im Bereich des Exons 11 und 15 [30]. BRAF-V600E-Mutationen treten oftmals isoliert auf, Non-V600Mutationen sind hingegen häufig mit weiteren onkogenen Mutationen, wie beispielsweise im EGFR- oder KRASGen assoziiert [14].

\section{Klinische Besonderheiten}

Generell weisen Patienten mit BRAFMutationen dieselben Charakteristika (Alter, Geschlecht, Rasse, Raucherstatus, Stadium) auf wie Patienten ohne onkogene Treibermutation. Die Subgruppe der V600-Mutation tritt jedoch häufiger bei Leicht- /Nichtrauchern auf und geht häufiger mit intrathorakalen Metastasen einher, während bei Non-V600-Mutationen intraabdominelle Metastasen häufiger sind [24].

\section{Molekulare Diagnostik}

Der Nachweis von BRAF-Mutationen kann durch DNA-basierte Methoden (Multiplex-Primer-Extension-basierte Methoden oder „next-generation se-

chemie für die häufigste Mutation (BRAF V600E) erfolgen.

\section{Therapie}

Im Vergleich $\mathrm{zu}$ anderen zielgerichteten Therapien waren die therapeutischen Erfolge einer BRAF-Blockade mit einer Ansprechrate von $33 \%$ und einer Dauer des Ansprechens von 9,6 Monaten zunächst eher enttäuschend. In 2 Studien konnten mit der Kombination des MEK-Inhibitors Trametinib mit dem BRAF-Inhibitor Dabrafenib eine Ansprechrate von 63\% bei Patienten mit BRAF-V600E-mutiertem Adenokarzinom und ein medianes Überleben von 24,6 Monaten bei unvorbehandelten Patienten erreicht werden [35].

Retrospektive Analysen des klinischen Verlaufs von Patienten mit BRAFmutiertem NSCLC ergaben ein heterogenes Bild in Bezug auf das mediane Gesamtüberleben mit Werten zwischen 15,2 und 56,5 Monaten [22]. Eine groBe Kohorte umfasste 72 Patienten mit $B R A F-$ Mutationen. Davon erhielten nur 20 Patienten einen BRAF/MEK-Inhibitor in der zweiten Therapielinie oder später.
Das mediane OS betrug 56,5 (95\%-KI: 13,4-89,1) Monate im Vergleich zu 27,2 $(10,6-64,6)$ Monaten bei Patienten, die keine zielgerichtete Therapie erhalten hatten [15]. In der French Cooperative Thoracic Intergroup Biomarkers France Study fanden sich bei 83 Patienten mit $B R A F-$ Mutationen, von denen nur $21 \mathrm{mit}$ einen BRAF/MEK-Inhibitor behandelt wurden, keine Unterschiede bezüglich PFS und „disease control rate“ (DCR) [7].

Die molekulare Stratifizierung des NSCLC schreitet weiter voran, und es werden weitere molekulare Treiber, auch solche, die für Resistenzen verantwortlich gemacht werden, in Studien untersucht. Zum Teil sind auch schon Behandlungsoptionen zugelassen. Einige onkogene Treiber sind in - Tab. 3 zusammengefasst.

\section{Fazit für die Praxis}

- Die Subgruppe der Patienten mit nichtkleinzelligem Lungenkarzinom (NSCLC) und behandelbaren Treibermutationen profitiert von einer zielgerichteten Therapie.
- Die molekulare Diagnostik ist Basis für die Therapieplanung in der Erstlinientherapie.

- Rebiopsie oder "liquid biopsy“ sind erforderlich, um Resistenzmechanismen aufzuspüren und zielgerichtet weiterzubehandeln.

- Für EGFR-mutierte Tumoren sind Tyrosinkinaseinhibitoren (TKI) der 1. (Erlotinib, Gefitinib), 2. (Afatinib, Dacomitinib) und 3. Generation (Osimertinib) zugelassen.

- Die Kombination TKI und VEGF-Inhibitor bzw. TKI und Chemotherapie stellt möglicherweise eine Verbesserung dar.

- Für Tumoren mit EML4/ALK-Translokation sind Crizotinib, Ceritinib und Alectinib für die Erstlinientherapie, Brigatinib nach Crizotinibversagen und Lorlatinib nach Versagen der ersten TKI-Therapie zugelassen.

- Für Tumoren mit ROS1-Translokation, BRAF-Mutation, NTRK-Fusionen, HER2-Mutation, MET-Exon-14-Skipping stehen zielgerichtete Substan- 
zen zur Verfügung, die z.T. schon zugelassen sind.

\section{Korrespondenzadresse}

\section{Prof. Dr. Susanne M. Lang}

Klinik für Innere Medizin II, Sektion Pneumologische Onkologie, Universitätsklinikum Jena

Am Klinikum 1, 07747 Jena, Deutschland

susanne.lang@med.uni-jena.de

Funding. Open Access funding enabled and organized by Projekt DEAL.

\section{Einhaltung ethischer Richtlinien}

Interessenkonflikt. S. Lang gibt an, Honorare für Vortragstätigkeit, Beteiligung an klinischen Studien, Mitarbeit in einem Wissenschaftlichen Beirat von AstraZeneca, BMS, Boehringer, Lilly, MSD, Novartis, Pfizer und Roche erhalten zu haben. T. Rachow gibt an, Honorare für Vortragstätigkeit, Beteiligung an klinischen Studien von BMS und Pfizer erhalten zu haben.

Für diesen Beitrag wurden von den Autoren keine Studien an Menschen oder Tieren durchgeführt. Für die aufgeführten Studien gelten die jeweils dort angegebenen ethischen Richtlinien.

Open Access. Dieser Artikel wird unter der Creative Commons Namensnennung 4.0 International Lizenz veröffentlicht, welche die Nutzung, Vervielfältigung Bearbeitung, Verbreitung und Wiedergabe in jeglichem Medium und Format erlaubt, sofern Sie den/die ursprünglichen Autor(en) und die Quelle ordnungsgemäß nennen, einen Link zur Creative Commons Lizenz beifügen und angeben, ob Änderungen vorgenommen wurden.

Die in diesem Artikel enthaltenen Bilder und sonstiges Drittmaterial unterliegen ebenfalls der genannten Creative Commons Lizenz, sofern sich aus der Abbildungslegende nichts anderes ergibt. Sofern das betreffende Material nicht unter der genannten Creative Commons Lizenz steht und die betreffende Handlung nicht nach gesetzlichen Vorschriften erlaubt ist, ist für die oben aufgeführten Weiterverwendungen des Materials die Einwilligung des jeweiligen Rechteinhabers einzuholen.

\section{Das erste theragnostische Tandem für GEP-NET}

Theragnostik ist der neue Weg in der Nuklearmedizin: Zielgerichtete nuklearmedizinische Therapie und Diagnostik können mit demselben Target kombiniert werden. Bei gastroenteropankreatischen neuroendokrinen Tumoren (GEPNET) kommt dieser Behandlungsansatz bereits zum Einsatz. Weitere Indikationen, zum Beispiel das Prostata-Karzinom, werden in den nächsten Jahren folgen.

Ursprünglich für die präzise diagnostische Bildgebung in Form von Szintigraphie, Positronen-Emissionstomographie (PET, in Verbindung mit CT und MRT) und Einzelphotonen-Emissionscomputertomographie (SPECT) eingesetzt, rückten in den vergangenen Jahren nuklearmedizinische Therapien wie die Peptidrezeptor-Radionuklidtherapie (PRRT) in den Fokus der Forschung und Anwendung. „Bei Somatostatinrezeptor-positiven Tumoren findet die PRRT ab der Zweitlinie Anwendung", so Prof. Dr. Marianne Pavel vom Universitätsklinikum Erlangen in einem online-Expertengespräch von Advanced Accelerator Applications - AAA. Die Wirksamkeit der PRRT basiere auf der Tumorbiologie der NET. Deshalb gelte es jeweils individuell abzuwägen, welche Patienten zu welchem Zeitpunkt der Behandlung von einer PRRT profitieren. Bei Dünndarm-NET sei die PRRT bereits jetzt die häufigste Option der Zweitlinientherapie.

NETTER-1 zeigt Wirkung

Die erste Phase-III-Studie mit der Bezeichnung NETTER-1, die Wirksamkeit und Sicherheit von ${ }^{177} \mathrm{Lu}$-Dotatate, eines mit ${ }^{177} \mathrm{Lu}$ markierten Somatostatin-Analog-Peptids, bei Patienten mit gut differenzierten (G1 und G2) GEP-NET untersuchte, zeigte, ein signifikant verlängertes Progressionsfreies Überleben (PFS) für Patienten unter einer ${ }^{177}$ Lu-Dotatate-Therapie im Vergleich zu einer Behandlung mit Somatostatin-Analoga (SSA). Dabei wurde das Risiko für einen Krankheitsfortschritt um 79 Prozent verringert. Als Meilenstein-Studie bezeichnete Prof. Dr. Markus Essler, Direktor der Klinik und Poliklinik für Nuklearmedizin des Universitätsklinikums Bonn, NETTER-1, die den Durchbruch der theragnostischen Nuklearmedizin bedeute. Sie habe evidenz basiert aufgezeigt, wie gering die Nebenwirkungen in dieser Patientengruppe sind. Die Langzeitwirkung müsse nun noch untersucht werden. „PRRT ist eine Therapie für viele, aber nicht für alle." Für die richtige Patientenauswahl bedarf es großer Expertise, betonte auch Pavel. Hier komme dem Mehrwert eines interdisziplinären Teams im Tumorboard große Bedeutung zu.

Ein Spin off des CERN

Mit SomaKit TOC ${ }^{\mathrm{TM}}$, einem Kit zur Synthese einer ${ }^{68} \mathrm{Ga}$-Edotreotidlösung für die PET, und dem PRRT-Arzneimittel Lutathera bietet das Unternehmen Advanced Accelerator Applications - AAA das erste zugelassene theragnostische Tandem zur Diagnose beziehungsweise Behandlung von gut differenzierten (G1 oder G2) Somatostatinrezeptor-positiven GEP-NET an. AAA ist ursprünglich ein Spin-off des CERN in Genf und seit 2018 100 prozentiges Tochterunternehmen von Novartis.

Quelle: Presseinformation Advanced Accelerator Applications - AAA

\section{Originalpublikation:}

NETTER-1: Strosberg J et al. Phase 3 Trial of 177Lu-Dotatate for Midgut Neuroendocrine Tumors. N EnglJ Med 2017;376:125-135. 
Onkologie

Weitere Details zur Lizenz entnehmen Sie bitte der Lizenzinformation auf http://creativecommons.org/ licenses/by/4.0/deed.de.

\section{Literatur}

1. Aisner DL, Sholl LM, Berry LD et al (2018) The impact of smoking and TP53 mutations in lung adenocarcinoma patients with targetable mutations - the Lung Cancer Mutation Consortium (LCMC2). Clin Cancer Res 24:1038-1047

2. Griesinger F, EberhardtW, Früh M, Gautschi $O$, Hilbe W, Hoffmann H, Huber RM, Loges S, Pirker R, Pöttgen C, Pritzkuleit R, Reck M, Reinmuth N, Sebastian M, Ukena D, Waller C, Wolf J, Wolf M, Wörmann B (2019) Onkopedia Leitlinie nicht-kleinzelliges Lungenkarzinom. https://www.onkopedia.com/de/ onkopedia/guidelines/lungenkarzinom-nichtkleinzellig-nsclc/@@guideline/html/index.html. Zugegriffen: 28.05.2020

3. Arcila ME, Drilon A, Sylvester BE et al (2015) MAP2K1 (MEK1) mutations define a distinct subset of lung adenocarcinoma associated with smoking. Clin Cancer Res 21:1935-1943

4. Bar-Sagi D, Knelson EH, Sequist LV (2020) A bright future for KRAS inhibitors. Nat Cancer 1:25-27

5. Chia PL, Mitchell P, Dobrovic A et al (2014) Prevalence and natural history of ALK positive non-small-cell lung cancer and the clinical impact of targeted therapy with ALK inhibitors. Clin Epidemiol 6:423-432

6. Chiari R, Ricciuti B, Landi L et al (2020) ROS1-rearranged non-small-cell lung cancer is associated with a high rate of venous thromboembolism: analysis from a phase II, prospective, multicenter, twoarms trial (METROS). Clin Lung Cancer 21:15-20

7. Couraud S, Barlesi F, Fontaine-Deraluelle $C$ et al (2019) Clinical outcomes of non-small-cell lung cancer patients with BRAF mutations: results from the French Cooperative Thoracic Intergroup biomarkers France study. Eur J Cancer 116:86-97

8. Digumarthy SR, Mendoza DP, Lin JJ et al (2020) Computed tomography imaging features and distribution of metastases in ROS1-rearranged non-small-cell lung cancer. Clin Lung Cancer 21(2):153-159.e3

9. Dong ZY, Zhang JT, Liu SY et al (2017) EGFR mutation correlates with uninflamed phenotype and weak immunogenicity, causing impaired response to PD-1 blockade in non-small cell lung cancer. Oncolmmunology 6:e1356145

10. Drilon A, Clark JW, Weiss J et al (2020) Antitumor activity of crizotinib in lung cancers harboring a MET exon 14 alteration. Nat Med 26:47-51

11. Gainor JF, Dardaei L, Yoda S et al (2016) Molecular mechanisms of resistance to first- and secondgeneration ALK inhibitors in ALK-rearranged lung cancer. Cancer Discov 6:1118-1133

12. Goss G, Tsai CM, Shepherd FA et al (2016) Osimertinib for pretreated EGFR Thr790Metpositive advanced non-small-cell lung cancer (AURA2): a multicentre, open-label, single-arm, phase 2 study. Lancet Oncol 17:1643-1652

13. Graham RP, Treece AL, Lindeman NI et al (2018) Worldwide frequency of commonly detected EGFR mutations. Arch Pathol Lab Med 142:163-167

14. Guo Y, Cao R, Zhang X et al (2019) Recent progress in rare oncogenic drivers and targeted therapy for non-small cell lung cancer. Onco Targets Ther 12:10343-10360

15. Horn L, Bauml J, Forde PM et al (2019) Real-world treatment patterns and survival of patients with
BRAF V600-mutated metastatic non-small cell lung cancer. Lung Cancer 128:74-90

16. Hosomi Y, Morita S, Sugawara S et al (2020) Gefitinib alone versus gefitinib plus chemotherapy for non-small-cell lung cancer with mutated epidermal growth factor receptor: NEJ009 study. JClin Oncol 38:115-123

17. Jia Y, Yun CH, Park E et al (2016) Overcoming EGFR(T790M) and EGFR(C797S) resistance with mutant-selective allosteric inhibitors. Nature 534:129-132

18. Kalemkerian GP, Narula N, Kennedy EB et al (2018) Molecular testing guideline for the selection of patients with lung cancer for treatment with targeted tyrosine kinase inhibitors: American Society of Clinical Oncology Endorsement of the College of American Pathologists/International Association for the Study of Lung Cancer/ Association for Molecular Pathology clinical practice guideline update. J Clin Oncol 36:911-919

19. Katayama R, Friboulet L, Koike S et al (2014) Two novel ALK mutations mediate acquired resistance to the next-generation ALK inhibitor alectinib. Clin Cancer Res 20:5686-5696

20. Leitlinienprogramm Onkologie (2018) S3-Leitlinie Prävention, Diagnostik, Therapie und Nachsorge des Lungenkarzinoms. AWMF-Registernummer: 020/007OL. http://leitlinienprogrammonkologie.de/Lungenkarzinom.98.0.html. Zugegriffen:28.05.2020

21. Lee CK, Man J, Lord S et al (2018) Clinical and molecular characteristics associated with survival among patients treated with checkpoint inhibitors for advanced non-small cell lung carcinoma: a systematic review and meta-analysis. JAMA Oncol 4:210-216

22. Marchetti A, Felicioni L, Malatesta $S$ et al (2011) Clinical features and outcome of patients with nonsmall-cell lung cancer harboring BRAF mutations. JClin Oncol 29:3574-3579

23. Mazieres J, Zalcman G, Crino Let al (2015) Crizotinib therapy for advanced lung adenocarcinoma and a ROS1 rearrangement: results from the EUROS1 cohort. J Clin Oncol 33:992-999

24. Mendoza DP, Dagogo-Jack I, Chen T et al (2019) Imaging characteristics of BRAF-mutant non-small cell lung cancer by functional class. Lung Cancer 129:80-84

25. Mok TS, Cheng Y, Zhou Xet al (2018) Improvement in overall survival in a randomized study that compared dacomitinib with gefitinib in patients with advanced non-small-cell lung cancer and EGFRactivating mutations. JClin Oncol 36:2244-2250

26. Nakagawa K, Garon EB, Seto T et al (2019) Ramucirumab plus erlotinib in patients with untreated, EGFR-mutated, advanced non-smallcell lung cancer (RELAY): a randomised, doubleblind, placebo-controlled, phase 3 trial. Lancet Oncol 20:1655-1669

27. Noronha V, Patil VM, Joshi A et al (2020) Gefitinib versus gefitinib plus pemetrexed and carboplatin chemotherapy in EGFR-mutated lung cancer.J $\mathrm{Clin}$ Oncol 38:124-136

28. Oscorbin IP, Shadrina AS, Kozlov VV et al (2019) Absence of EGFR C797S mutation in tyrosine kinase inhibitor-naive non-small cell lung cancer tissues. Pathol Oncol Res. https://doi.org/10.1007/ s12253-019-00683-4

29. Oxnard GR, Thress KS, Alden RS et al (2016) Association between plasma genotyping and outcomes of treatment with osimertinib (AZD9291) in advanced non-small-cell lung cancer. J Clin Oncol 34:3375-3382
30. Paik PK, Arcila ME, Fara M et al (2011) Clinical characteristics of patients with lung adenocarcinomas harboring BRAF mutations. J Clin Oncol 29:2046-2051

31. Pao W, Miller VA, Politi KA et al (2005) Acquired resistance of lung adenocarcinomas to gefitinib or erlotinib is associated with a second mutation in the EGFR kinase domain. PLoSMed 2:e73

32. ParkK, Tan EH, O'Byrne Ketal (2016) Afatinibversus gefitinib as first-line treatment of patients with EGFRmutation-positivenon-small-cell lung cancer (LUX-Lung 7): a phase 2B, open-label, randomised controlled trial. Lancet Oncol 17:577-589

33. Peters S, Camidge DR, Shaw AT etal (2017) Alectinib versus crizotinib in untreated ALK-positive nonsmall-cell lung cancer. N Engl J Med 377:829-838

34. Pillai RN, Behera M, Berry LD et al (2017) HER2 mutations in lung adenocarcinomas: a report from the Lung Cancer Mutation Consortium. Cancer 123:4099-4105

35. Planchard D, Besse B, Groen HJM et al (2016) Dabrafenib plus trametinib in patients with previously treated BRAF(V600E)-mutant metastatic nonsmall cell lung cancer: an open-label, multicentre phase 2 trial. Lancet Oncol 17:984-993

36. Planchard D, Popat S, Kerr K et al (2019) Metastatic non-small cell lung cancer: ESMO Clinical Practice Guidelines for diagnosis, treatment and follow-up. Ann Oncol 30:863-870

37. Ramalingam SS, Vansteenkiste J, Planchard D et al (2020) Overall survival with osimertinib in untreated, EGFR-mutated advanced NSCLC.NEngl JMed 382:41-50

38. Rangachari $D$, Yamaguchi $N$, Vanderlaan $P A$ et a (2015) Brain metastases in patients with EGFRmutated or ALK-rearranged non-small-cell lung cancers. Lung Cancer 88:108-111

39. Reck M, Mok TSK, Nishio M et al (2019) Atezolizumab plus bevacizumab and chemotherapy in non-small-cell lung cancer (IMpower150): key subgroup analyses of patients with EGFR mutations or baseline liver metastases in a randomised, openlabel phase 3 trial. Lancet Respir Med 7:387-401

40. Shaw AT, Felip E, Bauer TM et al (2017) Lorlatinib in non-small-cell lung cancer with ALK or ROS1 rearrangement: an international, multicentre, open-label, single-arm first-in-man phase 1 trial. Lancet Oncol 18:1590-1599

41. Shaw AT, Kim DW, Nakagawa K et al (2013) Crizotinib versus chemotherapy in advanced ALKpositive lung cancer. NEngl J Med 368:2385-2394

42. Soda M, Choi YL, Enomoto M et al (2007) Identification of the transforming EML4-ALK fusion gene in non-small-cell lung cancer. Nature 448:561-566

43. Solomon BJ, Mok T, Kim DW et al (2014) First-line crizotinib versus chemotherapy in ALK-positive lung cancer. N Engl J Med 371:2167-2177

44. Zer A, Moskovitz M, Hwang DM et al (2017) ALKrearranged non-small-cell lung cancer is associated with a high rate of venous thromboembolism. Clin Lung Cancer 18:156-161

45. Zhang T, Wan B, Zhao Y et al (2019) Treatment of uncommon EGFR mutations in non-small cell lung cancer: new evidence and treatment. Transl Lung Cancer Res 8:302-316 